\title{
Sparse Beamforming Design for Network MIMO System with Per-Base-Station Backhaul Constraints
}

\author{
Binbin Dai and Wei Yu \\ Department of Electrical and Computer Engineering \\ University of Toronto, Toronto, Ontario M5S 3G4, Canada \\ Emails: \{bdai,weiyu\}@comm.utoronto.ca
}

\begin{abstract}
This paper considers the joint beamforming and clustering design problem in a downlink network multiple-input multiple-output (MIMO) setup, where the base-stations (BSs) are connected to a central processor with rate-limited backhaul links. We formulate the problem as that of devising a sparse beamforming vector across the BSs for each user, where the nonzero beamforming entries correspond to that user's serving BSs. Differing from the previous works, this paper explicitly formulates the per-BS backhaul constraints in the network utility maximization framework. In contrast to the traditional utility maximization problem with transmit power constraint only, the additional backhaul constraints result in a discrete $\ell_{0}$ norm formulation, which makes the problem more challenging. Motivated by the compressive sensing literature, we propose to iteratively approximate the per-BS backhaul constraints using a reweighted $\ell_{1}$-norm technique and reformulate the backhaul constraints as weighted per-BS power constraints. This allows us to solve the weighted sum rate maximization problem through a generalized weighted minimum mean square error (WMMSE) approach. To reduce the computational complexity of the proposed algorithm within each iteration, we propose two additional techniques, iterative link removal and iterative user pool shrinking, which dynamically decrease the potential BS cluster size and user scheduling pool. Numerical results show that the proposed algorithm can significantly improve the system throughput as compared to the naive BS clustering strategy based on the channel strength.
\end{abstract}

\section{INTRODUCTION}

Network multiple-input multiple-output (MIMO) is a promising technique for improving the system performance of future wireless cellular networks. In practice, network MIMO system can be realized by connecting the base-stations (BSs) to a central processor (CP) with high-speed backhaul links. The ideal implementation of network MIMO system, where all the BSs fully cooperate and each BS acquires the channel state information (CSI) of the entire network, requires very high backhaul capacities and is not practical. With finite-rate backhaul links, the CP can share each user's message with a limited set of BSs (called BS cluster in this paper), which then cooperatively serve the user through joint beamforming.

To determine the optimal BS clustering and joint beamforming strategies for the downlink network MIMO system with limited backhaul is not an easy task. First, as each user wants to be served by the strongest BSs while each BS has a limit on the number of users it can support, the optimal BS clustering design involves a tradeoff between the user rates and the backhaul capacities. The naive cluster selection scheme purely based on the channel strength may lead to severely imbalanced traffic load, especially in heterogeneous networks where the macro-BSs usually have much higher transmit power than the pico-BSs. Second, even with fixed BS clustering, finding the optimal joint beamforming is also nontrivial since different clusters for different users may overlap.

In this paper, we propose to jointly address the above two issues by devising a sparse beamforming strategy across the BSs for each user, where the nonzero beamforming entries correspond to that user's serving BSs. In contrast to [1] [4] which formulate the problem as radio resources minimization under fixed user rate constraints, this paper focuses on network utility maximization under given radio resources, namely transmit power and backhaul capacities. We propose an efficient algorithm to select the BS cluster for each user dynamically and to design the corresponding beamforming vector according to the given backhaul limits.

Utility maximization for the network MIMO system considered in existing literature mostly takes the limited backhaul capacities into account implicitly by either fixing the BS clusters [5]-[7] or adding the backhaul as a penalized term into the objective function [8]-[10]. Specifically, [5] considers sum rate maximization under fixed and non-overlapping clustering scheme while [6] and [7] maximize a more general utility function under potentially overlapping but predetermined BS clusters. Dynamic clustering design is considered in [8] by penalizing the objective function with an $\ell_{2}$-norm approximation of the cluster size. Alternatively, [9] and [10] choose the backhaul rate as the penalized term but solve the problem heuristically. For fixed clustering scheme assumed in [5] [7], the backhaul consumption is only known afterwards by evaluating the rates of user messages delivered in each backhaul link. For dynamic clustering designs considered in [8]-[10], one has to optimally choose the price associated with each penalized term to ensure that the overall backhaul stays within the budget, which is not easy.

In contrast to all the above existing works, the main contribution of this paper is that we explicitly account for the per-BS backhaul constraints in the overall network utility maximization framework. Differing from the utility maximization problems in conventional wireless networks with only power constraints, the additional backhaul constraints in network MIMO system make the problem more challenging. The backhaul consumption at a particular BS is a function 


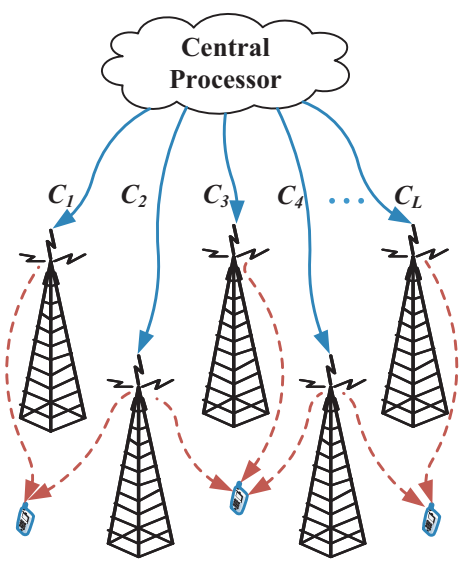

Fig. 1: Network MIMO with per-BS backhaul constraints.

of not only the (continuous) user rates but also the (discrete) number of associated users.

Motivated by the compressive sensing literature [11], this paper casts the BS clustering problem as an $\ell_{0}$-norm optimization problem and proposes to iteratively approximate the mixed continuous and discrete per-BS backhaul rate through an $\ell_{1}$-norm reweighting technique, which essentially reformulates the per-BS backhaul constraints into weighted perBS power constraints. This novel approximation allows us to apply a generalized weighted minimum mean square error (WMMSE) approach [7] to solve the utility maximization problem. The resulting algorithm efficiently determines the optimal BS clustering and beamforming strategies jointly for the network MIMO system with limited backhaul.

In order to further improve the efficiency of the proposed algorithm, we propose two additional techniques, iterative link removal and iterative user pool shrinking, which iteratively exclude those BSs with negligible transmit power out of the candidate cluster set for each user, and exclude those users with negligible rates out of the user scheduling pool, respectively. Simulation results show that the proposed algorithm can fully utilize the limited backhaul resource and significantly improve the system throughput as compared to the conventional user-centric clustering scheme based on channel strength.

\section{System Model AND PRoblem Formulation}

Consider a downlink cellular network with $L$ BSs and $K$ users, where each BS has $M$ transmit antennas while each user has $N$ receive antennas. Each BS $l$ is connected to a $\mathrm{CP}$ via a backhaul link with capacity limit $C_{l}, l=1,2, \cdots, L$, as depicted in Fig. 1. With linear transmit beamforming scheme, the received signal at user $k$, denoted as $\mathbf{y}_{k} \in \mathbb{C}^{N \times 1}, k=$ $1,2, \cdots, K$, can be modeled as

$$
\mathbf{y}_{k}=\mathbf{H}_{k} \mathbf{w}_{k} s_{k}+\sum_{j \neq k} \mathbf{H}_{k} \mathbf{w}_{j} s_{j}+\mathbf{n}_{k},
$$

where $\mathbf{H}_{k} \in \mathbb{C}^{N \times M_{t}}$ and $\mathbf{w}_{k} \in \mathbb{C}^{M_{t} \times 1}=\left[\mathbf{w}_{k}^{1}, \mathbf{w}_{k}^{2}, \cdots, \mathbf{w}_{k}^{L}\right]$ denote the CSI matrix and beamforming vector respectively from all the $M_{t}=L M$ transmit antennas ${ }^{1}$ to user $k$. Suppose BS $l$ is not part of user $k$ 's serving cluster, then the corresponding beamforming entries $\mathbf{w}_{k}^{l} \in \mathbb{C}^{M \times 1}$ are set to 0 . In this paper, we consider the case where each user has only a single data stream for simplicity and assume that user $k$ 's message $s_{k} \in \mathbb{C}$ is independent and identically distributed according to $\mathcal{C N}(0,1)$. Here, $\mathbf{n}_{k} \in \mathbb{C}^{N \times 1}$ is the received noise at user $k$ and modeled as $\mathbf{n}_{k} \sim \mathcal{C N}\left(0, \sigma^{2} \mathbf{I}\right)$.

We assume that the CP has access to all the users' data and has global CSI for designing the optimal sparse beamforming vector $\mathbf{w}_{k}$. Once $\mathbf{w}_{k}$ is determined, the CP transmits user $k$ 's message, along with the beamforming coefficients, to those BSs corresponding to the nonzero entries in $\mathbf{w}_{k}$ through the backhaul links. In this paper, we assume that the channels are slow varying and only consider the backhaul consumption due to the user data sharing and ignore the backhaul required for sharing CSI and delivering beamforming coefficients. Under this assumption, the per-BS backhaul constraint can be cast in the following weighted $\ell_{0}$-norm format:

$$
\sum_{k}\|\| \mathbf{w}_{k}^{l}\left\|_{2}^{2}\right\|_{0} R_{k} \leq C_{l}, \quad \forall l
$$

where $R_{k}$ is the achievable rate for user $k$ defined as

$$
\begin{aligned}
& R_{k}= \\
& \log \left(1+\mathbf{w}_{k}^{H} \mathbf{H}_{k}^{H}\left(\sum_{j \neq k} \mathbf{H}_{k} \mathbf{w}_{j} \mathbf{w}_{j}^{H} \mathbf{H}_{k}^{H}+\sigma^{2} \mathbf{I}\right)^{-1} \mathbf{H}_{k} \mathbf{w}_{k}\right)
\end{aligned}
$$

Intuitively, the backhaul consumption at the $l$ th $\mathrm{BS}$ is the accumulated data rates of the users served by BS $l$. Here, \|\| $\mathbf{w}_{k}^{l}\left\|_{2}^{2}\right\|_{0}$ characterizes whether or not BS $l$ serves user $k$ :

$$
\|\| \mathbf{w}_{k}^{l}\left\|_{2}^{2}\right\|_{0}=\left\{\begin{array}{ll}
0, & \text { if }\left\|\mathbf{w}_{k}^{l}\right\|_{2}^{2}=0 \\
1, & \text { otherwise }
\end{array} .\right.
$$

This paper considers network utility maximization as the objective. Among the family of utility functions, weighted sum rate (WSR) has been widely applied to network control and optimization problems. In this paper, we also adopt the WSR utility but point out that the proposed scheme can be readily extend to any utility function that holds an equivalence relationship with the WMMSE minimization problem (see [12] for a sufficient condition on the utility functions holding such an equivalence).

With per-BS power and per-BS backhaul constraints, the WSR maximization problem can be formulated as:

$$
\begin{array}{ll}
\underset{\left\{\mathbf{w}_{k}^{l}\right\}}{\operatorname{maximize}} & \sum_{k} \alpha_{k} R_{k} \\
\text { subject to } & \sum_{k}\left\|\mathbf{w}_{k}^{l}\right\|_{2}^{2} \leq P_{l}, \forall l \\
& \sum_{k}\|\| \mathbf{w}_{k}^{l}\left\|_{2}^{2}\right\|_{0} R_{k} \leq C_{l}, \quad \forall l
\end{array}
$$

${ }^{1}$ To simplify the notations, we assume that all the $L$ BSs can potentially serve each scheduled user. In practice, only the strongest few BSs around each user need to be considered as the candidate serving BSs. 
where $\alpha_{k}$ denotes the priority weight associated with user $k$, $P_{l}$ and $C_{l}$ represent the transmit power budget and backhaul capacity limit for BS $l$, respectively.

\section{Proposed Algorithm}

Conventional WSR maximization problem is a well-known nonconvex problem, for which finding the global optimality is already quite challenging even without the additional backhaul constraint. This paper therefore focuses on solving for the local optimum solution of the problem (5) only. Our main contribution is a new way of dealing with the discrete $\ell_{0}$-norm constraint $(5 \mathrm{c})$.

In compressive sensing literature, nonconvex $\ell_{0}$-norm objective is often approximated by the convex reweighted $\ell_{1}$-norm [11]. In this paper, we extend this idea to the $\ell_{0}$-norm in the constraint and approximate $(5 \mathrm{c})$ as

$$
\sum_{k} \beta_{k}^{l} R_{k}\left\|\mathbf{w}_{k}^{l}\right\|_{2}^{2} \leq C_{l}
$$

where $\beta_{k}^{l}$ is a constant weight associated with BS $l$ and user $k$ and is updated iteratively according to

$$
\beta_{k}^{l}=\frac{1}{\left\|\mathbf{w}_{k}^{l}\right\|_{2}^{2}+\tau}, \forall k, l
$$

with some small constant regularization factor $\tau>0$ and $\left\|\mathbf{w}_{k}^{l}\right\|_{2}^{2}$ from the previous iteration.

Even with the above approximation, the optimization problem (5) with the backhaul constraint (5c) replaced by (6) is still difficult to deal with due to the fact that the rate $R_{k}$ in the constraint is unknown. To address this difficulty, we propose to solve the problem (5) iteratively with fixed rate $\hat{R}_{k}$ in (6) and update $\hat{R}_{k}$ by the achievable rate $R_{k}$ from the previous iteration. Under fixed $\beta_{k}^{l}$ and $\hat{R}_{k}$, problem (5) now reduces to

$$
\begin{array}{ll}
\underset{\left\{\mathbf{w}_{k}^{l}\right\}}{\operatorname{maximize}} & \sum_{k} \alpha_{k} R_{k} \\
\text { subject to } & \sum_{k}\left\|\mathbf{w}_{k}^{l}\right\|_{2}^{2} \leq P_{l}, \forall l \\
& \sum_{k} \beta_{k}^{l} \hat{R}_{k}\left\|\mathbf{w}_{k}^{l}\right\|_{2}^{2} \leq C_{l}, \quad \forall l
\end{array}
$$

where the approximated backhaul constraint (8c) can be interpreted as a weighted per-BS power constraint bearing a resemblance to the traditional per-BS power constraint (8b).

Although the approximated problem (8) is still nonconvex, we can reformulate it as an equivalent WMMSE minimization problem in order to reach a local optimum solution. The equivalence between WSR maximization and WMMSE minimization is first established in [13] for MIMO broadcast channel and later generalized to MIMO interfering channel in [12] and MIMO interfering channel with partial cooperation in [7]. It is not difficult to see that the generalized WMMSE equivalence established in [12] also extends to the problem (8) with the newly introduced weighted per-BS power constraint (8c). We explicitly state the equivalence as follows:
Proposition 3.1 ( [12]): The WSR maximization problem (8) has the same optimal solution with the following WMMSE minimization problem:

$$
\begin{array}{ll}
\underset{\left\{\rho_{k}, \mathbf{u}_{k}, \mathbf{w}_{k}^{l}\right\}}{\operatorname{minimize}} & \sum_{k} \alpha_{k}\left(\rho_{k} e_{k}-\log \rho_{k}\right) \\
\text { subject to } & \sum_{k}\left\|\mathbf{w}_{k}^{l}\right\|_{2}^{2} \leq P_{l}, \forall l \\
& \sum_{k} \beta_{k}^{l} \hat{R}_{k}\left\|\mathbf{w}_{k}^{l}\right\|_{2}^{2} \leq C_{l}, \forall l
\end{array}
$$

where $\rho_{k}$ denotes the MSE weight for user $k$ and $e_{k}$ is the corresponding MSE defined as

$$
\begin{aligned}
& e_{k}=\mathrm{E}\left[\left\|\mathbf{u}_{k}^{H} \mathbf{y}_{k}-s_{k}\right\|_{2}^{2}\right] \\
& =\mathbf{u}_{k}^{H}\left(\sum_{j} \mathbf{H}_{k} \mathbf{w}_{j} \mathbf{w}_{j}^{H} \mathbf{H}_{k}^{H}+\sigma^{2} \mathbf{I}\right) \mathbf{u}_{k}-2 \operatorname{Re}\left\{\mathbf{u}_{k}^{H} \mathbf{H}_{k} \mathbf{w}_{k}\right\}+1
\end{aligned}
$$

under receiver $\mathbf{u}_{k} \in \mathbb{C}^{N \times 1}$.

The advantage of solving the WSR maximization problem (8) through its equivalent WMMSE minimization problem (9) is that (9) is convex with respect to each of the individual optimization variables. This crucial observation allows the problem (9) to be solved efficiently through the block coordinate descent method by iterating between $\rho_{k}, \mathbf{u}_{k}$ and $\mathbf{w}_{k}$ :

- The optimal MSE weight $\rho_{k}$ under fixed $\mathbf{w}_{k}$ and $\mathbf{u}_{k}$ is $\rho_{k}=e_{k}^{-1}, \forall k$.

- The optimal receiver $\mathbf{u}_{k}$ under fixed $\mathbf{w}_{k}$ and $\rho_{k}$ is the MMSE receiver:

$$
\mathbf{u}_{k}=\left(\sum_{j} \mathbf{H}_{k} \mathbf{w}_{j} \mathbf{w}_{j}^{H} \mathbf{H}_{k}^{H}+\sigma^{2} \mathbf{I}\right)^{-1} \mathbf{H}_{k} \mathbf{w}_{k}, \forall k .
$$

- The optimization problem to find the optimal transmit beamformer $\mathbf{w}_{k}$ under fixed $\mathbf{u}_{k}$ and $\rho_{k}$ is a quadratically constrained quadratic programming (QCQP) problem, which can be solved using standard convex optimization solvers such as CVX [14].

A straightforward but computationally intensive way of applying the above WMMSE algorithm to solve the original problem (5) would involve two loops: an inner loop to solve the approximated WSR maximization problem (8) with fixed weight $\beta_{k}^{l}$ and rate $\hat{R}_{k}$, and an outer loop to update $\beta_{k}^{l}$ and $\hat{R}_{k}$. Instead, we propose to combine these two loops into a single loop and update the weight $\beta_{k}^{l}$ and rate $\hat{R}_{k}$ inside the WMMSE algorithm, as summarized in Algorithm 1.

Algorithm 1 has the same complexity order as the conventional WMMSE algorithm since it only introduces two additional steps 4 and 5 in each iteration in updating $\beta_{k}^{l}$ and $\hat{R}_{k}$, which are both closed-form functions of the transmit beamformers. The main computational complexity of Algorithm 1 comes from the optimal transmit beamformer design in Step 3, which is a QCQP problem as mentioned before, but can also be equivalently reformulated as a second order cone programming (SOCP) problem as we do in the simulation part 


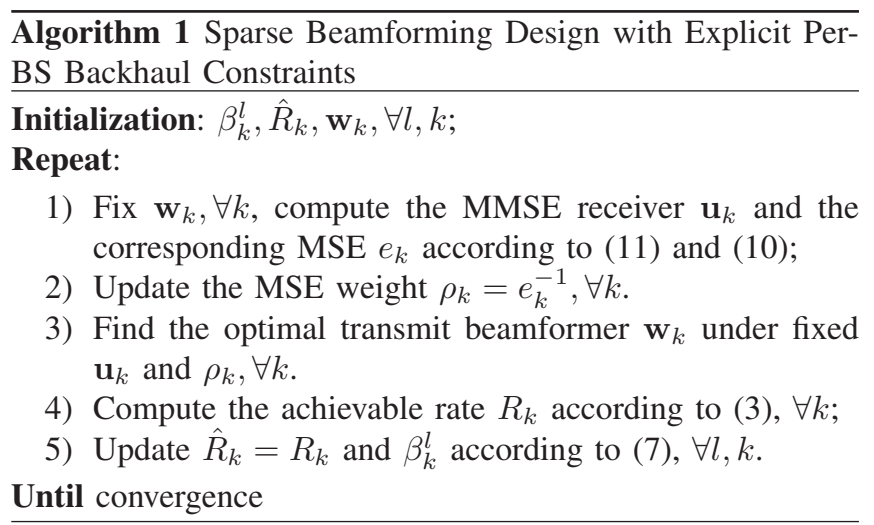

of this paper. The complexity of solving SOCP using interiorpoint method is approximately $\mathcal{O}\left((K L M)^{3}\right)$ [15].

To improve the efficiency of Algorithm 1 in each iteration, in what follows, we further propose two techniques, iterative link removal and iterative user pool shrinking. The former aims at reducing the number of potential transmit antennas $L M$ serving each user while the latter is intended to decrease the total number of users $K$ to be considered in each iteration.

\section{A. Iterative Link Removal}

Similar to what we observed in [2], the transmit power from some of the candidate serving BSs would drop down rapidly close to zero as the iterations go on. By taking advantage of this, we propose to iteratively remove the $l$ th BS from the $k$ th user's candidate cluster once the transmit power from BS $l$ to user $k$, i.e. $\left\|\mathbf{w}_{k}^{l}\right\|_{2}^{2}$, is below a certain threshold, say $-100 \mathrm{dBm} / \mathrm{Hz}$. This reduces the dimension of the potential transmit beamformer for each user and reduces the complexity of solving SOCP in Step 3 of Algorithm 1.

\section{B. Iterative User Pool Shrinking}

The WMMSE algorithm does user scheduling implicitly. We observe from simulations that, it is beneficial for Algorithm 1 to consider a large pool of users in the iterative process. However, to consider all the users in the entire network all the time would incur significant computational burden. Instead, we propose to check the achievable user rate $R_{k}$ in Step 4 iteratively and ignore those users with negligible rates (below some threshold, say $0.01 \mathrm{bps} / \mathrm{Hz}$ ) during the next iteration. Our simulations show that, after around 10 iterations, more than half of the total users can be taken out of the consideration with negligible performance loss to the overall algorithm. This significantly reduces the total number of variables to be optimized during the following iterations.

\section{Simulation Results}

In this section, numerical simulations are conducted to show the effectiveness of the proposed algorithm. We consider a 7-cell wrapped around two-tier heterogeneous network with the simulation parameters listed in Table I. Each cell forms a regular hexagon with a single macro-BS located in the center and 3 pico-BSs equally separated within the cell as
TABLE I: Simulation Parameters.

\begin{tabular}{|c|c|}
\hline $\begin{array}{c}\text { Cellular } \\
\text { Layout }\end{array}$ & $\begin{array}{c}\text { Hexagonal } \\
\text { 7-cell wrapped-around }\end{array}$ \\
\hline Channel bandwidth & $10 \mathrm{MHz}$ \\
\hline Distance between cells & $0.8 \mathrm{~km}$ \\
\hline Num. of (macro-BSs, pico-BSs, users)/cell & $(1,3,30)$ \\
\hline Num. of antennas/(macro-BS, pico-BS, user) & $(4,2,2)$ \\
\hline Max. Tx power for (macro-BS, pico-BS) & $(43,30) \mathrm{dBm}$ \\
\hline Antenna gain & $15 \mathrm{dBi}$ \\
\hline Background noise & $-169 \mathrm{dBm} / \mathrm{Hz}$ \\
\hline Path loss from macro-BS to user & $128.1+37.6 \log _{10}(d)$ \\
\hline Path loss from pico-BS to user & $140.7+36.7 \log _{10}(d)$ \\
\hline Log-normal shadowing & $8 \mathrm{~dB}$ \\
\hline Rayleigh small scale fading & $0 \mathrm{~dB}$ \\
\hline Reweighting function parameter & $\tau=10^{-10}$ \\
\hline
\end{tabular}

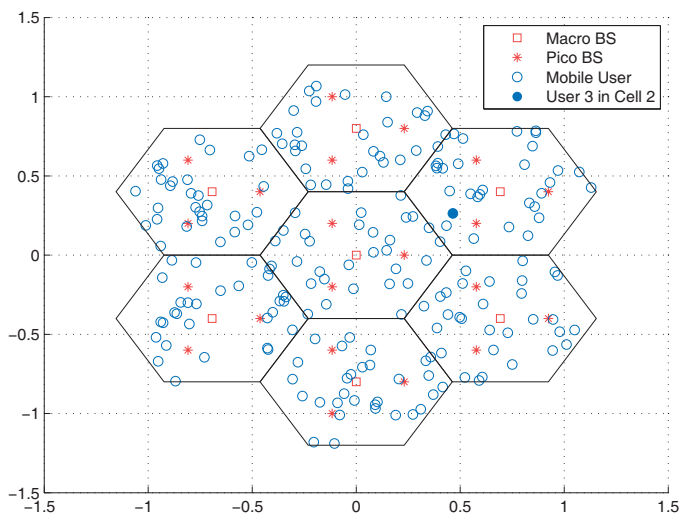

Fig. 2: 7-cell wrapped around two-tier heterogeneous network.

illustrated in Fig. 2. To simplify the discussion, we set all the macro-BSs to have equal backhaul constraints and likewise for the pico-BSs. The backhaul constraints are denoted as $\left(C_{\text {macro }}, C_{\text {pico }}\right)$ respectively. The proposed algorithm is simulated under the power constraints listed in Table I with various sets of $\left(C_{\text {macro }}, C_{\text {pico }}\right)$ backhaul constraints.

As indicated previously, instead of considering all the $L$ BSs in the entire network as the candidates serving each user, in simulations we only consider the strongest $L_{c}\left(L_{c} \leq L\right)$ $\mathrm{BSs}^{2}$ around each user as its candidate cluster. To illustrate how the sparse beamforming vector is formed in the proposed algorithm, we plot in Fig. 3 the power evolutions of the strongest $8 \mathrm{BSs}$ for the third user in the second cell as an example, without removing any BS out of the candidate cluster using the proposed iterative link removal technique. As we can see, after around 20 iterations only the first and third strongest BSs maintain at a reasonable transmit power level. They eventually form the cluster for serving user 3 in cell 2. With the proposed iterative link removal technique and by setting the threshold to be $-100 \mathrm{dBm} / \mathrm{Hz}$, the improved version of Algorithm 1 can narrow down the candidate BSs to only the strongest $4 \mathrm{BSs}$ after the 5th iteration, and the (1st, 3rd, 4th) strongest BSs after the 8th iteration, and finally the (1st, 3rd) strongest BSs after the 17th iteration.

\footnotetext{
${ }^{2}$ The strength of a BS seen by a user is evaluated by the maximum transmit power from that BS compensated with the path-loss to the user.
} 


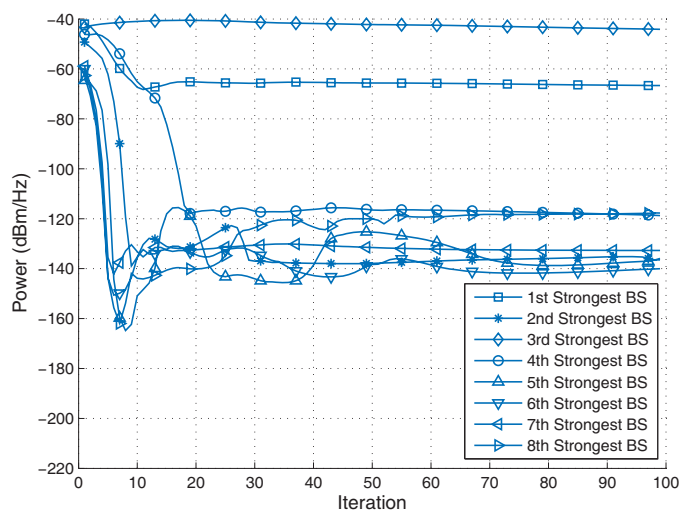

Fig. 3: Power evolutions of the strongest 8 BSs for user 3 in cell 2, $\left(C_{\text {macro }}, C_{\text {pico }}\right)=(245,70) \mathrm{Mbps}, \alpha_{k}=1, \forall k, L_{c}=8$.

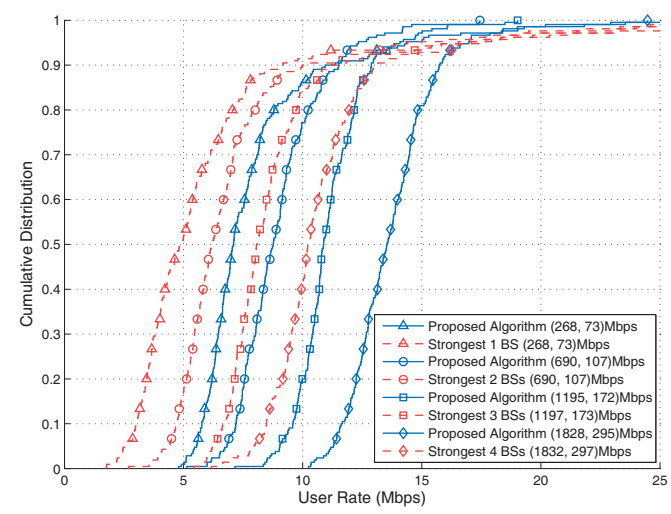

Fig. 4: User rates comparison with $L_{c}=8$ and $\alpha_{k}$ updated according to proportional fairness criterion.

In Fig. 4, we compare the cumulative distributions of the long-term average user rates between the proposed algorithm and the user-centric clustering scheme where each user is served by the strongest $S$ BSs $(S=1,2,3,4)$. We first run the baseline "Strongest $S$ BSs" schemes then evaluate the corresponding backhaul requirement for each BS afterwards. For the proposed algorithm, the explicit backhaul constraints $\left(C_{\text {macro }}, C_{\text {pico }}\right)$ are set to be the average backhaul over the macro-BSs and the pico-BSs respectively from the baseline. Each curve in Fig. 4 is obtained by iteratively simulating the corresponding scheme with fixed user priority weights $\alpha_{k}$ 's and updating the weights according to the proportional fairness criterion. As we can see, by optimizing with explicit backhaul constraints, the proposed algorithm achieves significant performance gain. For instance, around 35\% improvement is obtained for the 50th-percentile user as compared with the baseline where each user is served by the strongest 3 BSs. Note that since the baseline algorithm connects each user to an equal number of neighboring BSs, it inevitably favors high-rate users. In contrast, the proposed algorithm can select clusters for each user adaptively, and in particular can choose a larger cluster for the low-rate users, thus achieving a significant overall gain from a network utility perspective.

\section{CONCLUSION}

This paper proposes a novel $\ell_{1}$-norm reweighting technique for solving the utility maximization problem for a network MIMO system with limited per-BS backhaul capacities. The proposed algorithm dynamically selects the BS cluster and designs the corresponding transmit beamformer for each user in each time slot according to the given backhaul constraints. To further reduce the computational complexity of the proposed algorithm, we propose to iteratively remove those BSs with negligible transmit power out of the candidate cluster for each user, and remove those users with negligible rates out of the user scheduling pool. Simulation results show that by explicitly accounting for the backhaul constraints into the utility maximization framework, the proposed algorithm can fully utilize the backhaul resource and significantly improve the system throughput as compared to the conventional usercentric cluster scheme purely based on the channel strength.

\section{REFERENCES}

[1] J. Zhao, T. Quek, and Z. Lei, "Coordinated multipoint transmission with limited backhaul data transfer," IEEE Trans. Wireless Commun., vol. 12, no. 6, pp. 2762-2775, 2013.

[2] B. Dai and W. Yu, "Sparse beamforming for limited-backhaul network MIMO system via reweighted power minimization," in Proc. IEEE Global Telecommunications Conf. (Globecom), 2013.

[3] F. Zhuang and V. Lau, "Backhaul limited asymmetric cooperation for MIMO cellular networks via semidefinite relaxation," IEEE Trans. Signal Process., vol. 62, no. 3, pp. 684-693, Feb. 2014.

[4] S. Luo, R. Zhang, and T. J. Lim, "Downlink and uplink energy minimization through user association and beamforming in cloud RAN," 2014. [Online]. Available: http://arxiv.org/abs/1402.4238

[5] J. Zhang, R. Chen, J. Andrews, A. Ghosh, and R. Heath, "Networked MIMO with clustered linear precoding," IEEE Trans. Wireless Commun., vol. 8, no. 4, pp. 1910-1921, 2009.

[6] C. Ng and H. Huang, "Linear precoding in cooperative MIMO cellular networks with limited coordination clusters," IEEE J. Sel. Areas Commun., vol. 28, no. 9, pp. 1446-1454, 2010.

[7] S. Kaviani, O. Simeone, W. Krzymien, and S. Shamai, "Linear precoding and equalization for network MIMO with partial cooperation," IEEE Trans. Veh. Technol., vol. 61, no. 5, pp. 2083-2096, 2012.

[8] M. Hong, R.-Y. Sun, H. Baligh, and Z.-Q. Luo, "Joint base station clustering and beamformer design for partial coordinated transmission in heterogenous networks," IEEE J. Sel. Areas Commun., vol. 31, no. 2, pp. 226-240, Feb. 2013.

[9] A. Chowdhery, W. Yu, and J. Cioffi, "Cooperative wireless multicell OFDMA network with backhaul capacity constraints," in Proc. IEEE Int. Conf. Commun. (ICC), June 2011, pp. 1-6.

[10] S. Mehryar, A. Chowdhery, and W. Yu, "Dynamic cooperation link selection for network MIMO systems with limited backhaul capacity," in Proc. IEEE Int. Conf. Commun. (ICC), 2012.

[11] E. Candes, M. Wakin, and S. Boyd, "Enhancing sparsity by reweighted $\ell_{1}$ minimization," Journal of Fourier Analysis and Applications, vol. 14, no. 5, pp. 877-905, 2008

[12] Q. Shi, M. Razaviyayn, Z.-Q. Luo, and C. He, "An iteratively weighted MMSE approach to distributed sum-utility maximization for a MIMO interfering broadcast channel," IEEE Trans. Signal Process., vol. 59, no. 9, pp. $4331-4340$, Sept. 2011.

[13] S. Christensen, R. Agarwal, E. Carvalho, and J. Cioffi, "Weighted sumrate maximization using weighted MMSE for MIMO-BC beamforming design," IEEE Trans. Wireless Commun., vol. 7, no. 12, pp. 4792 -4799, Dec. 2008.

[14] M. Grant and S. Boyd, "CVX: Matlab software for disciplined convex programming, version 2.0 beta," Sept. 2013. [Online]. Available: http://cvxr.com/cvx

[15] Y. Ye, Interior Point Algorithms: Theory and Analysis. John Wiley \& Sons, 1997. 